\title{
Nonlinear OIFS for a Hybrid Galerkin Atmospheric Model
}

\author{
Amik St.-Cyr and Stephen J. Thomas \\ National Center for Atmospheric Research, \\ 1850 Table Mesa Drive, Boulder, 80305 CO, USA \\ \{amik, thomas\}@ucar.edu
}

\begin{abstract}
The purpose of this paper is to explore a time-split hybrid Galerkin scheme for the atmospheric shallow water equations. A nonlinear variant of operator integration factor splitting is employed as the time-stepping scheme. The hyperbolic system representing slow modes is discretized using the discontinuous Galerkin method. An implicit second order backward differentiation formula is applied to Coriolis and gravity wave terms. The implicit system is then discretised using a continuous Galerkin spectral element method. The advantages of such an approach include improved mass and energy conservation properties. A strong-stability preserving Runge-Kutta scheme is applied for substepping.
\end{abstract}

\section{Introduction}

The seminal work of Robert (1981) led to a six-fold increase over the explicit time step for atmospheric general circulation models. To achieve such dramatic gains without recourse to a fully implicit integrator, a semi-Lagrangian treatment of advection was combined with a semi-implicit scheme for the stiff terms responsible for gravity waves. Initially, semi-implicit semi-Lagrangian time-stepping was applied to hyperbolic problems, discretized using low-order finite-differences and finite elements. The traditional semi-Lagrangian algorithm implemented in atmospheric models relies on backward trajectory integration and upstream interpolation, Staniforth and Côté (1991). A potentially lower cost alternative is the operator integrating factor splitting (OIFS) method of Maday et al. (1990) which relies on Eulerian sub-stepping of the advection equation. In contrast with semiLagrangian advection, there are no dissipation or dispersion errors associated with upstream interpolation or trajectory integration and the scheme maintains the high-order accuracy of the discrete spatial operators.

A discontinuous Galerkin shallow water model employing a nodal basis and explicit time-stepping is described in Giraldo et al. (2003). Sherwin (2004) demonstrated the advantages of a hybrid Galerkin approach in the context of the incompressible Navier-Stokes equations. Eskilsson and Sherwin (2005) describe a discontinuous Galerkin formulation of the shallow water equations using thirdorder strong-stability preserving (SSP) Runge-Kutta time-stepping. Here, we 
investigate a time-split scheme applied to the global shallow water equations in curvilinear coordinates on the cubed-sphere. A second order backward differentiation formula (BDF-2) is combined with SSP-RK sub-stepping of a hyperbolic system. Because the incompressibility constraint has been removed, the fully nonlinear OIFS scheme of St-Cyr and Thomas (2004) is employed. When compared to continuous Galerkin spectral elements, the hybrid scheme results in improved mass and energy conservation properties.

\section{Shallow Water Equations}

The shallow water equations contain the essential wave propagation mechanisms found in atmospheric general circulation models. These are the fast gravity waves and slow synoptic scale Rossby waves. The latter are important for correctly capturing nonlinear atmospheric dynamics. The governing equations of motion for the inviscid flow of a free surface are

$$
\begin{array}{r}
\frac{\partial \mathbf{v}}{\partial t}+(f+\zeta) \mathbf{k} \times \mathbf{v}+\frac{1}{2} \nabla(\mathbf{v} \cdot \mathbf{v})+\nabla \Phi=0 \\
\frac{\partial \Phi}{\partial t}+(\mathbf{v} \cdot \nabla) \Phi+\left(\Phi_{0}+\Phi\right) \nabla \cdot \mathbf{v}=0 .
\end{array}
$$

$h$ is the height above sea level, $\mathbf{v}$ is the horizontal velocity and $\Phi=g h$ the geopotential height. $f$ is the Coriolis parameter and $\mathbf{k}$ a unit vector in the vertical direction. The geopotential height is decomposed into a perturbation about a constant base state, $\Phi_{0}$.

To exploit the potential of operator integration factor splitting for systems of time-dependent partial differential equations, St.-Cyr and Thomas (2004) show that a fully nonlinear form of the algorithm is more appropriate. Sub-stepping is applied to

$$
\begin{aligned}
\frac{\partial \tilde{\mathbf{v}}}{\partial s}+\tilde{\zeta} \mathbf{k} \times \tilde{\mathbf{v}}+\frac{1}{2} \nabla(\tilde{\mathbf{v}} \cdot \tilde{\mathbf{v}}) & =0 \\
\frac{\partial \tilde{\Phi}}{\partial s}+\nabla \cdot(\tilde{\Phi} \tilde{\mathbf{v}}) & =0 .
\end{aligned}
$$

with initial conditions $\tilde{\mathbf{v}}\left(\mathbf{x}, t^{n-q}\right)=\mathbf{v}\left(\mathbf{x}, t^{n-q}\right), \tilde{\Phi}\left(\mathbf{x}, t^{n-q}\right)=\Phi\left(\mathbf{x}, t^{n-q}\right)$. The integration factor $Q_{S}^{t^{*}}(t)$ is applied to the remaining de-coupled system of equations containing the Coriolis and linear gravity wave terms

$$
\frac{d}{d t} Q_{S}^{t^{*}}(t)\left[\begin{array}{c}
\mathbf{v} \\
\Phi
\end{array}\right]=-Q_{S}^{t^{*}}(t)\left[\begin{array}{c}
f \mathbf{k} \times \mathbf{v}+\nabla \Phi \\
\Phi_{0} \nabla \cdot \mathbf{v}
\end{array}\right]
$$

An accurate representation of fast-moving gravity waves is not required for large scale atmospheric dynamics and the corresponding terms can be treated implicitly. For a second order BDF-2 scheme, sub-stepping of the rhs terms is not required because $Q_{S}^{t^{n}}\left(t^{n}\right)=I$. 
The resulting time discretization of (5) is given by

$$
\begin{aligned}
\mathbf{v}^{n}+\frac{2}{3} \Delta t \mathbf{N} \nabla \Phi^{n} & =\frac{4}{3} \mathbf{N} \tilde{\mathbf{v}}^{n-1}-\frac{1}{3} \mathbf{N} \tilde{\mathbf{v}}^{n-2} \\
\Phi^{n}+\frac{2}{3} \Delta t \Phi_{0} \nabla \cdot \mathbf{v}^{n} & =\frac{4}{3} \tilde{\Phi}^{n-1}-\frac{1}{3} \tilde{\Phi}^{n-2}
\end{aligned}
$$

where

$$
\mathbf{N}=\left(I+\frac{2}{3} \Delta t f \mathbf{M}\right)^{-1}, \quad \mathbf{M}=\left[\begin{array}{rr}
0 & -1 \\
1 & 0
\end{array}\right]
$$

The values of the fields $\tilde{\mathbf{v}}$ and $\tilde{\Phi}$ at time levels $n-1$ and $n-2$ are computed by substepping (3) and (4) on the intervals $\left[t^{n-1}, t^{n}\right]$ and $\left[t^{n-2}, t^{n}\right]$. An implicit equation for $\Phi^{n}$ is obtained after space discretization and application of block Gaussian elimination, resulting in a modified Helmholtz problem. The coefficient matrix of this linear system of equations is non-symmetric due to the implicit treatment of the Coriolis terms and is solved using an iterative conjugate-gradient squared (CGS) algorithm with a block-Jacobi preconditioner.

For high-order spectral elements, under-integration using Gaussian quadrature results in discrete operators where the eigenvalues corresponding to high frequency modes are shifted into the right-half plane. A filter is therefore required to stabilize the time-stepping scheme for long time integrations, Fischer and Mullen (2001). Strong-stability preserving (SSP) time integration schemes maintain the non-oscillatory properties of the spatial operator, Gottlieb et al (2001). We next describe the computational domain, high-order Galerkin finite element approximations and SSP-RK sub-stepping.

\section{High-Order Galerkin Approximations}

The flux form shallow-water equations in curvilinear coordinates are described in Sadourny (1972). Let $\mathbf{a}_{1}$ and $\mathbf{a}_{2}$ be the covariant base vectors of the transformation between inscribed cube and spherical surface. The metric tensor is defined as $G_{i j} \equiv \mathbf{a}_{i} \cdot \mathbf{a}_{j}$. Covariant and contravariant vectors are related through the metric tensor by $u_{i}=G_{i j} u^{j}, u^{i}=G^{i j} u_{j}$, where $G^{i j}=\left(G_{i j}\right)^{-1}$ and $G=\operatorname{det}\left(G_{i j}\right)$. The six local coordinate systems $\left(x^{1}, x^{2}\right)$ are based on equiangular central projection, $-\pi / 4 \leq x^{1}, x^{2} \leq \pi / 4$.

$$
G_{i j}=\frac{1}{r^{4} \cos ^{2} x^{1} \cos ^{2} x^{2}}\left[\begin{array}{cc}
1+\tan ^{2} x^{1} & -\tan x^{1} \tan x^{2} \\
-\tan x^{1} \tan x^{2} & 1+\tan ^{2} x^{2}
\end{array}\right]
$$

where $r=\left(1+\tan ^{2} x^{1}+\tan ^{2} x^{2}\right)^{1 / 2}$ and $\sqrt{G}=1 / r^{3} \cos ^{2} x^{1} \cos ^{2} x^{2}$. 
The time-split hyperbolic system $(3)-(4)$ can be written in as

$$
\begin{aligned}
\frac{\partial u_{1}}{\partial t}+\frac{\partial}{\partial x^{1}} E & =\sqrt{G} u^{2}(f+\zeta), \\
\frac{\partial u_{2}}{\partial t}+\frac{\partial}{\partial x^{2}} E & =-\sqrt{G} u^{1}(f+\zeta), \\
\frac{\partial}{\partial t}(\sqrt{G} \Phi)+\frac{\partial}{\partial x^{1}}\left(\sqrt{G} u^{1} \Phi\right)+\frac{\partial}{\partial x^{2}}\left(\sqrt{G} u^{2} \Phi\right) & =0,
\end{aligned}
$$

where

$$
E=\frac{1}{2}\left(u_{1} u^{1}+u_{2} u^{2}\right), \quad \zeta=\frac{1}{\sqrt{G}}\left[\frac{\partial u_{2}}{\partial x^{1}}-\frac{\partial u_{1}}{\partial x^{2}}\right]
$$

Consider a scalar component of the hyperbolic system,

$$
\frac{\partial u}{\partial t}+\nabla \cdot \mathcal{F}=S
$$

The computational domain $\Omega$ is partitioned into elements $\Omega_{k}$. An approximate solution $u_{h}$ belongs to the finite dimensional space $\mathcal{V}_{h}(\Omega)$. Multiplication of by a test function $\varphi_{h} \in \mathcal{V}_{h}$ and integration over the element $\Omega_{k}$ results in a weak Galerkin formulation of the problem.

$$
\frac{\partial}{\partial t} \int_{\Omega_{k}} \varphi_{h} u_{h} d \Omega=\int_{\Omega_{k}} \varphi_{h} S d \Omega+\int_{\Omega_{k}} \mathcal{F} \cdot \nabla \varphi_{h} d \Omega-\int_{\partial \Omega_{k}} \varphi_{h} \mathcal{F} \cdot \hat{n} d s .
$$

For a discontinuous Galerkin approximation, a nodal basis for $\mathcal{V}_{h}$ is employed, consisting of the Legendre cardinal functions. The solutions $u_{h}$ are expanded in terms of tensor-product basis functions on a Gauss-Lobatto grid.

The flux function $\mathcal{F} \cdot \hat{n}$ is approximated by a Lax-Friedrichs numerical flux

$$
\widehat{\mathcal{F}}\left(u_{h}^{+}, u_{h}^{-}\right)=\frac{1}{2}\left[\left(\mathcal{F}\left(u_{h}^{+}\right)+\mathcal{F}\left(u_{h}^{-}\right)\right) \cdot \hat{n}-\alpha\left(u_{h}^{+}-u_{h}^{-}\right)\right] .
$$

Boundary integrals are computed using higher-order Gaussian quadrature. $\alpha$ is the upper bound for the absolute value of eigenvalues of the flux Jacobian $\mathcal{F}^{\prime}(u)$ in the direction $\hat{n}$. Equations $(9)-(11)$ are written in the semi-discrete form

$$
\frac{d \mathbf{u}}{d t}=\mathbf{L}(\mathbf{u}) \text {. }
$$

The second-order three stage SSP RK2-3 integrator of Higueras (2004) is applied to sub-step the above system of ordinary differential equations. This scheme has a CFL number $C=2$ and efficiency factor $C / 3=2 / 3$. The implicit system (6) - (7) is discretized using $\mathbb{P}_{N}-\mathbb{P}_{N}$ continuous Galerkin spectral elements.

\section{Numerical Experiments}

Our numerical experiments are based on the shallow water test suite of Williamson et al (1992). Test case 5 is a zonal flow impinging on an isolated 
mountain. The center of the mountain is located at $(3 \pi / 2, \pi / 6)$ with height $h_{s}=$ $2000(1-r / R)$ meters, where $R=\pi / 9$ and $r^{2}=\min \left[R^{2},(\lambda-3 \pi / 2)^{2}+(\theta-\pi / 6)^{2}\right]$. Initial wind and height fields are

$$
\begin{aligned}
u & =u_{0}\left(\cos \alpha_{0} \cos \theta+\sin \alpha_{0} \cos \lambda \sin \theta\right) \\
v & =-u_{0} \sin \alpha_{0} \sin \lambda \\
g h & =g h_{0}-\frac{u_{0}}{2}\left(2 a \Omega+u_{0}\right)\left(\sin \theta \cos \alpha_{0}-\cos \lambda \cos \theta \sin \alpha_{0}\right)^{2}
\end{aligned}
$$

$a$ is the earth's radius, $\Omega$ the rotation rate, $\alpha_{0}=0, g h_{0}=5960 \mathrm{~m}^{2} / \mathrm{s}^{2}$ and $u_{0}=20 \mathrm{~m} / \mathrm{s}$. The geopotential height field after 15 days of integration is plotted in Fig. 1. The solution is smooth and does not exhibit spurious oscillations.

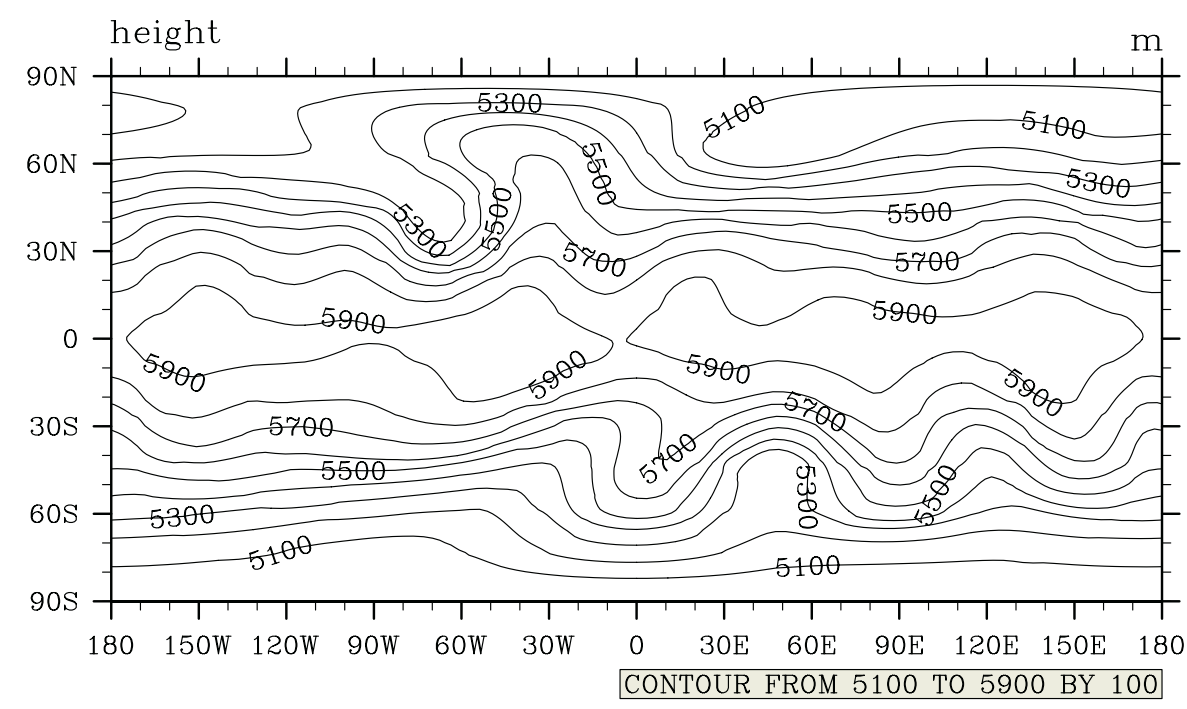

Fig. 1. Shallow water test case 5: Flow impinging on a mountain. Geopotential height field $h$ at fifteen days produced by hybrid scheme. 150 spectral elements, $8 \times 8$ GaussLegendre Lobatto points per element

The implementation of the BDF-2/RK2-3 scheme is still at an experimental stage. However, it leads to an increase in the model integration rate. Figure 2 is a plot of the ratio of $\mathrm{CNLF}$ and $\mathrm{BDF}-2 / \mathrm{RK} 2-3$ execution times for test case 5 . The efficiency gain of BDF-2/RK2-3 over the CNLF scheme is close to a factor of two. Thomas and Loft (2002) observed that the CNLF scheme integrated twice as fast as an explicit spectral element shallow water model. Therefore, the total increase in the model integration rate, when compared to the explicit model, is a factor of four. The plateau in the efficiency curve is due to a growing number of solver iterations as the time step size increases. 


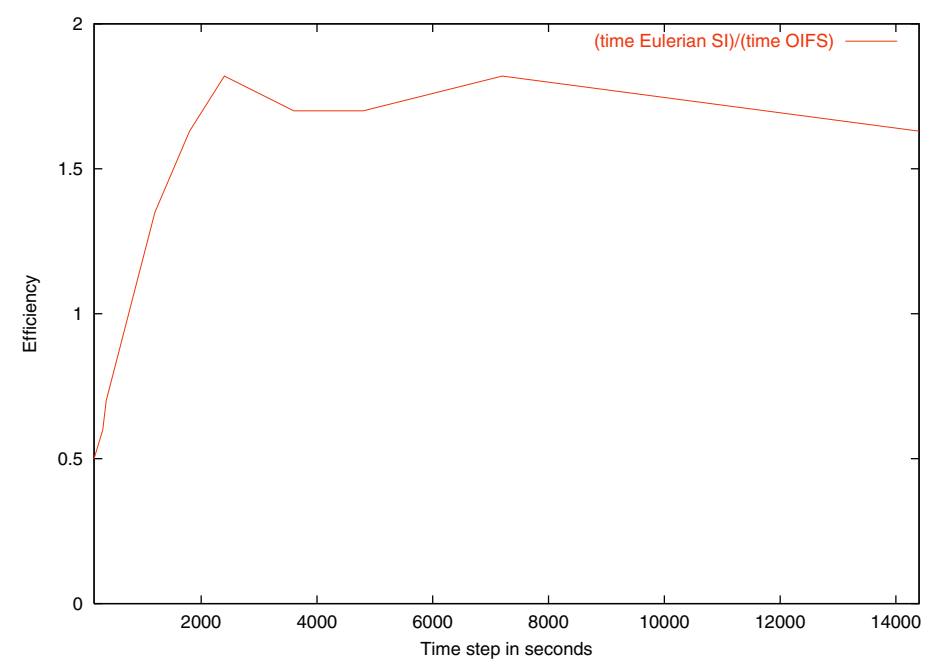

Fig. 2. Shallow water test case 5: Flow impinging on a mountain. Computational efficiency. Ratio of the CNLF and BDF-2/RK2-3 model execution times. $K=150$ spectral elements, $8 \times 8$ Gauss-Legendre points per element

\section{Conclusions}

The larger time step permitted by the time-split BDF-2/RK2-3 integrator implies that the hybrid Galerkin discretization is well-suited for modeling multiscale atmospheric phenomena. The computation time is currently dominated by an increasing number of solver iterations as the time step size increases. However, a recently developed optimized Schwarz preconditioner maintains a much lower iteration count, St.-Cyr et al (2004). We estimate that this preconditioner could lead to an additional factor of four improvement in the computational efficiency compared to a Crank-Nicholson leap-frog (CNLF) formulation. Although the hybrid scheme is not strictly conservative, the mass loss is significantly less than in a continuous Galerkin spectral element model.

For parallel computation, a clear advantage of the discontinuous Galerkin method is the ability to overlap computation with communication. Specifically, the communication of conserved variables can be overlapped with the computation of the weak divergence and source terms. We are also currently experimenting with a hybrid MPI/OpenMP programming model for SMP cluster computer architectures and plan to report these results in the near future.

\section{References}

1. Eskilsson, C. and S. J. Sherwin, 2005: Discontinuous Galerkin spectral/hp element modelling of dispersive shallow water systems. J. Sci. Comp., 22, 279-298. 
2. Fischer, P. F. and J. S. Mullen, 2001: Filter-Based stabilization of spectral element methods. Comptes Rendus de l'Acadmie des sciences Paris, t. 332, Série I - Analyse numérique, 265-270.

3. Giraldo, F. X., J. S. Hesthaven, and T. Warburton, 2003: Nodal high-order discontinuous Galerkin methods for spherical shallow water equations. J. Comput. Phys., 181, 499-525.

4. Gottlieb, S., C. W. Shu, and E. Tadmor, 2001: Strong stability preserving highorder time discretization methods. SIAM Review, 43, 89-112.

5. Higueras, I., 2004: On strong stability preserving time discretization methods. $J$. Sci. Comput., 21, 193-223.

6. Maday, Y., A. T. Patera, and E. M. Ronquist, 1990: An operator-integration-factor splitting method for time-dependent problems: Application to incompressible fluid flow. J. Sci. Comput., 5, 263-292.

7. Robert, A. J., 1981: A stable numerical integration scheme for the primitive meteorological equations. Atmos.-Ocean, 19, 35-46.

8. Sadourny, R., 1972: Conservative finite-difference approximations of the primitive equations on quasi-uniform spherical grids. Mon. Wea. Rev., 100, 136-144.

9. Sherwin, S. J., 2002: A sub-stepping Navier-Stokes splitting scheme for spectral/hp element discretisations. Parallel Computational Fluid Dynamics, North Holland.

10. Staniforth, A., and J. Coté 1991: Semi-Lagrangian integration schemes for atmospheric models - A review. Mon. Wea. Rev., 119, 2206-223.

11. St-Cyr, A., and S. J. Thomas, 2004: Nonlinear operator integration factor splitting for the shallow water equations. Appl. Numer. Math., to appear.

12. St-Cyr, A., M. Gander and S. J. Thomas, 2004: Optimized RAS preconditioning. Proceedings of the 2004 Copper Mountain Conference on Iterative Methods.

13. Thomas, S. J., and R. D. Loft, 2002: Semi-implicit spectral element atmospheric model. J. Sci. Comp., 17, 339-350.

14. Williamson, D. L., J. B. Drake, J. J. hack, R. Jakob, P. N. Swarztrauber, 1992: A standard test set for numerical approximations to the shallow water equations in spherical geometry J. Comp. Phys., 102, 211-224. 